Gedankenguts des NS - Chefideologen Alfred Rosenberg in so manchen Urteilstext - wohl keine wirklich „furchtbaren Juristen“ in der Verwaltungsgerichtsbarkeit gegeben hat, wohl auch hauptsächlich deshalb, weil sie keine Zuständigkeit in wirklich brisanten Angelegenheiten hatte. Er hat aber auch einschränkend hinzugefügt, dass von einer gründlichen Aufarbeitung dieses Kapitels wohl noch nicht gesprochen werden könne, ${ }^{10}$ und an diesem Befund hat sich bis heute - wenn ich es recht sehe - nichts geändert.

\title{
7. Struktur \\ der Verwaltungsgerichtsbarkeit in der Bundesrepublik
}

Zur Überleitung auf die Zeit nach 1945 kann ich leider nicht mehr auf die Porträts der ersten Präsidenten des BVerwG verweisen, die hier bis zum Jahre 2002 an der Wand hingen, seither aber in Leipzig still aber konzentriert vor sich hin schimmern. So kann ich nur umstandslos darauf hinweisen, dass auch die restlichen Verwaltungsgerichte, soweit sie noch formal bestanden, 1945 durch Militärregierungsgesetz Nr.2 geschlossen wurden, aber bereits Ende 1946 durch Kontrollratsgesetz Nr. 36 in den westlichen Besatzungszonen wieder eingeführt wurden.

In der Bundesrepublik stellte dann das Grundgesetz die entscheidenden Weichen für den Zuschnitt der heutigen deutschen Verwaltungsgerichtsbarkeit. Die Zersplitterung des verwaltungsgerichtlichen Verfahrensrechts, die der Tatsache geschuldet war, dass die Verwaltungsgerichte zunächst in den verschiedenen Ländern und nach dem Krieg in den verschiedenen Besatzungszonen ihren Anfang nahm, wurde durch das Inkrafttreten der Verwaltungsgerichtsordnung im Jahre 1960 endgültig beseitigt.

10 Vgl. Sendler VBIBW 1989, 41, 45. 
Die Verwaltungsgerichtsbarkeit der Bundesrepublik wurde vor allem durch zwei Bestimmungen des Grundgesetzes geprägt:

Zum einen durch Art. 19 Abs. 4 GG, wonach jeder, der durch die öffentliche Gewalt in einem Recht verletzt wird, gerichtlichen Rechtsschutz in Anspruch nehmen kann. Also eine umfassende Generalklausel, die einen lückenlosen Rechtsschutz ermöglicht.

Zum anderen Art. 1 Abs. 3 GG, wonach die Grundrechte unmittelbare Bindungswirkung für Legislative, Exekutive und Judikative entfalten. Der stärkere Fokus auf die Grundrechte führte zu einer Sensibilisierung in der Rechtsauslegung und Rechtsanwendung - Stichworte wie „verfassungskonforme Rechtsauslegung“ oder die Anwendung des Grundsatzes der Verhältnismäßigkeit in allen Lebenslagen des Verwaltungsalltags weisen auf diese Sensibilisierung hin.

Der frühere Präsident des Bundesverwaltungsgerichts Werner hat diesen Sachverhalt prägnant bezeichnet mit dem berühmten Satz:

„Verwaltungsrecht ist konkretisiertes Verfassungsrecht“.

Der oben zitierte Satz Otto Meyers „Verfassungsrecht vergeht, Verwaltungsrecht besteht" ist damit weitgehend obsolet geworden.

Diese unmittelbare Grundrechtsbindung wird auch in der Metapher deutlich, das Bundesverwaltungsgericht sei der „kleine Bruder“ des Bundesverfassungsgerichts, oder die Verwaltungsgerichtsbarkeit die kleine Schwester - gegendert könnten wir uns auch auf das „kleinere Geschwister" einigen.

In diese Richtung gehen auch Formulierungen wie: Das Verfassungsgericht sei der Grundpfeiler des Rechtsstaats, die Eckpfeiler seien die Verwaltungsgerichte.

Mir ist diese Metaphorik mit der Großarchitektur von Säulen und Pfeilern nicht so ganz geheuer. Zum einen war mir als ehemaligem kleinen Ziegelstein eines Eckpfeilers gar nicht so recht bewusst, welch ungeheure Last ich zu tragen hatte - das ganze Haus des Rechtsstaats nämlich. Zum anderen scheint mir die Bedeutung der Verwaltungsgerichtsbarkeit dann doch etwas zu euphorisch dargestellt zu sein.

Trotz meines Hangs zu mehr Bescheidenheit wollte ich Ihnen diese Zitate nicht vorenthalten, und zwar vor allem aus didaktischen Gründen: Die bildhafte Übertreibung bringt oft einen größeren Erkenntnisgewinn als die ausgewogene Rede. 
Und der Erkenntnisgewinn soll sein:

Die umfassende Zuständigkeit und der verfassungsrechtlich erweiterte Prüfungsumfang haben in der Bundesrepublik zu einem Bedeutungszuwachs der Verwaltungsgerichtsbarkeit geführt, der sich auch rein äußerlich darin niederschlägt, dass wir heute eine dreistufige Gerichtsbarkeit haben (VG, OVG/VGH, BVerwG) gegenüber der früheren Einstufigkeit.

Diesem Bedeutungszuwachs steht - wenn man so will - inhaltlich eine gewisse Beschränkung gegenüber: Das letzte Wort in Verfassungsfragen hat das Bundesverfassungsgericht, dem man ja jedenfalls Wortfaulheit nicht nachsagen kann.

Eine ganz ähnliche Entwicklung (Bedeutungszuwachs bei gleichzeitiger inhaltlicher Beschränkung) ist auf dem Gebiet des Europarechts festzustellen. Die deutschen Richter im Allgemeinen und die Verwaltungsrichter im Besonderen haben in zunehmendem Maß Europarecht anzuwenden, sei es in Form nationaler Gesetze zur Umsetzung von EU-Richtlinien, sei es in Form von EU-Verordnungen. Insbesondere im Wirtschaftsverwaltungsrecht, im Planungsrecht, im Subventionsrecht und im Umweltrecht ist fast immer auch Europarecht im Spiel. Insoweit ist jeder deutsche Richter auch „Europarichter", der das nationale Recht europarechtskonform auszulegen und dabei auch den vom EuGH entwickelten effet utile zu berücksichtigen hat. Hat ein Richter der letzten Instanz Zweifel hinsichtlich einer europarechtlichen Frage, muss er diese in einem Vorabentscheidungsverfahren dem EuGH vorlegen.

Diese europarechtliche Einbindung besteht zwar seit gut 60 Jahren, wird aber auf der Gerichtsebene erst in den letzten etwa 20 Jahren ihrer Bedeutung entsprechend wahrgenommen. Noch zum 125-jährigen Bestehen der deutschen Verwaltungsgerichtsbarkeit hat etwa Horst Sendler in seinem damaligen Festvortrag die europarechtliche Ebene gar nicht erwähnt. Das war auch verständlich. Von Beginn der Statistik des EuGH im Jahr 1952 bis Ende 1980 gab es insgesamt nur neun Vorabentscheidungsersuchen durch das BVerwG. Inzwischen sind es etwa 100 Ersuchen, davon allein 50 in den letzten zehn Jahren. ${ }^{11}$ Der

11 von Danwitz NVwZ-Beilage 1/2013, 44. 
deutsche Richter am EuGH, von Danwitz, hat deshalb jüngst zu Recht von einem veritablen Rechtsprechungsdialog zwischen BVerwG und EuGH gesprochen.

Der Bedeutungszuwachs durch unmittelbare Prüfung von Verfassungs- und Europarecht weist übrigens auf ein Phänomen hin, das den Verwaltungsrichter tendenziell stärker betrifft als andere Gerichtsbarkeiten: Das Problem des sog. Mehr-Ebenen-Rechts. Eine Wasserabgabesatzung als Rechtssetzung einer kleinen Gemeinde beruht auf einer landesrechtlichen Ermächtigungsgrundlage, die sich im Rahmen einer bundesrechtlichen Regelung halten muss, die ihrerseits verfassungskonform sein muss und zudem eine EU-Richtlinie umzusetzen hatte.

Der Verwaltungsrichter muss sich hier also im Prinzip auf fünf Ebenen bewegen - ein akrobatischer Akt, der zwar nicht immer sturzfrei gelingt, sonst aber das wohlige Gefühl vermittelt, das manche nach dem erfolgreichen Lösen eines Kreuzworträtsels oder - aktueller - eines Sudokas haben mögen.

\section{Die Rechtsprechung als Spiegel der Zeitgeschichte}

Das eigentlich Spannende an der Verwaltungsgerichtsbarkeit ist aber, dass der Inhalt der Rechtsprechung als verlässlicher Spiegel der gerade aktuellen politischen und gesellschaftlichen Verhältnisse gelten kann. Ein kurzer Blick auf die Rechtsprechung nach 1945 bestätigt diesen Befund. In der unmittelbaren Nachkriegszeit hatte die Verbesserung der Versorgungssituation erste Priorität. So waren die Verwaltungsgerichte damals vor allem mit Fragen des Preis- und Flüchtlingsrechts, der Wohnraumbewirtschaftung und des Rechts der Heimkehrer befasst. Danach folgten zahlreiche Verfahren um Lastenausgleich und Besatzungsschäden. In den 50-er und 60-er Jahren rückten die bau- und abgaberechtlichen Fragen in den Vordergrund und spiegelten den Wiederaufbau und die ersten Erfolge des Wirtschaftsaufschwungs wider. Im Gefolge des Vietnamkriegs kam es Anfang der 70-er Jahre zu einer regelrechten Welle von Verfahren wegen Wehrdienstverweigerung. Die Reihe ließe 